\title{
MEASURES EQUIVALENT TO THE HAAR MEASURE
}

\author{
by $\mathrm{S}$. ŚWIERCZKOWSKI
}

(Received 19 November, 1959)

1. Introduction. We call two measures equivalent if each is absolutely continuous with respect to the other (cf. [1]). Let $G$ be a locally compact topological group and let $\mu$ be a nonnegative Baire measure on $G$ (i.e. $\mu$ is defined on all Baire sets, finite on compact sets and positive on open sets). We say that $\mu$ is stable if $\mu(E)=0$ implies $\mu(t E)=0$ for each $t \in G$. A. M. Macbeath made the conjecture that every stable non-trivial Baire measure is equivalent to the Haar measure. In this paper we prove the following slightly stronger result :

THeorem. Every stable non-trivial measure defined on Baire sets and finite on some open set is equivalent to the Haar measure.

It is obvious that not every stable measure on Baire sets is equivalent to the Haar measure; a counter-example is provided by an invariant Hausdorff measure in Euclidean space which is of lower dimension than the space itself.

Theorems B, C and Lemma 1 are due to A. M. Macbeath. He suggested to me the idea of constructing a Haar measure by means of a "Jacobian function ". We have used a similar method of proof in [2].

We assume now that $\mu$ is a stable non-trivial measure on Baire sets such that $\mu(U)<\infty$ for some open set $U$. Let us observe that $\mu(V)>0$ for every open set $V$. For, if $\mu(V)=0$, then $\mu(t V)=0$ and, since every compact set $C$ can be covered by a finite union of sets $t V$, we have $\mu(C)=0$ and the measure vanishes contrary to definition. Replacing, if necessary, $U$ by an open bounded subset, we may assume in the sequel that $U$ itself is bounded.

If $X$ is a topological space, we denote by $B(X)$ the class of all extended real-valued Baire functions $f(x)$ defined on $X$ (extended real numbers include $+\infty$ and $-\infty$ ) and by $B_{+}(X)$ the subclass of non-negative functions. We denote by $\mathbf{N}$ a complete system of bounded neighbourhoods of the unity $e$ of $G . \chi_{E}$ will be used to denote the characteristic function of the set $E$ (i.e. $\chi_{E}$ vanishes outside $E$ and is equal to 1 on $E$ ).

We have to show that if $E$ is a Baire set such that $\mu(E)=0$, then $E$ has Haar measure zero, and conversely. Since each Baire set $E$ is contained in a $\sigma$-compact $†$ open subgroup $G_{0}$ of $G$ (cf. [1], §5, Theorem D, p. 24) and the Haar measure on $G$ serves also as a Haar measure on $G_{0}$, and since moreover $\mu$ is stable on $G_{0}$, we may assume in the following that $G$ itself is $\sigma$-compact.

\section{Preliminary results.}

Theorem A. The measure $\mu$ is equivalent to a Baire measure $\bar{\mu}$.

Proof. Consider a set $T \subset G$ which is minimal with respect to the property

$$
G=\bigcup\{t U: t \in T\}
$$

(so that the family $\{t U\}, t \in T$, is a minimal covering of $G$ by sets $t U$ ). We show that every compact set $C$ intersects only a finite number of sets $t U, t \in T$. Suppose the contrary. The compact set $\overline{C U^{-1} U}$ can be covered by a subfamily $\{t U\}, t \in T^{*}$, where $T^{*}$ is a finite subset of $T$.

$\dagger$ A $\sigma$-compact set is a countable union of compact sets.

$\mathbf{L}$ 
On the other hand, by assumption, we have that $C \cap t U \neq \varnothing$ for infinitely many $t \in T$. Hence there is a $t_{0} \in T-T^{*}$ such that $C \cap t_{0} U \neq \varnothing$. We observe that $t_{0} U \subset C U^{-1} U$ and hence the family $\{t U\}, t \in T-\left\{t_{0}\right\}$, covers $G-C U^{-1} U$. It covers also $C U^{-1} U$ because $T^{*} \subset T^{\prime}-\left\{t_{0}\right\}$. This contradicts the minimal nature of $T$.

Since $G$ is $\sigma$-compact, $T$ is countable. We define

$$
\bar{\mu}(E)=\Sigma\left\{\mu\left(t^{-1} E \cap U\right): t \in T\right\} .
$$

Since, for fixed $t, \mu\left(t^{-1} E \cap U\right)$ is a Baire measure on the set $E$, we see that $\bar{\mu}$ is a Baire measure on $G$. Since $E$ is covered by the family $\{t U\}, t \in T$, we have that $\mu(E)=0$ is equivalent to $\mu(E \cap t U)=0$ for each $t \in T$. This last condition is equivalent to $\bar{\mu}(E)=0$, by the stability of $\mu$. Thus $\mu$ and $\bar{\mu}$ are equivalent. It is obvious that if $E$ is compact, then $\mu\left(t^{-1} E \cap U\right)>0$ holds only for a finite number of $t \in T$ and thus $\bar{\mu}(E)$ is finite. This completes the proof of the theorem.

By Theorem A, it is enough to prove our main result for $\bar{\mu}$ instead of for $\mu$. Equivalently, we shall assume that $\mu$ is a Baire measure.

Defrnitron. For given $t \in G$, a function $J_{t}(x) \in B_{+}(G)$ is called a $\mu$-Jacobian if, for every $f \in B_{+}(G)$,

$$
\int f\left(t^{-1} x\right) d \mu(x)=\int f(x) J_{t}(x) d \mu(x) .
$$

Theorem B. There exists, for every $t$, an everywhere positive $\mu$-Jacobian $J_{t}(x)$.

Proof. For a fixed $t$, define the measure $\mu_{t}$ on $G$ by $\mu_{t}(E)=\mu(t E)$. Since $\mu$ is stable, $\mu(E)=0$ is equivalent to $\mu_{t}(E)=0$ and thus the measures $\mu$ and $\mu_{t}$ are equivalent. Applying the Radon-Nikodym theorem to the totally $\sigma$-finite measures $\mu, \mu_{t}$ (cf. [1], $\S 31$, Theorem B, p. 128), we see that there is an everywhere positive function $J_{t}(x) \in B(G)$ with the property that

$$
\mu_{t}(E)=\int \chi_{E}\left(t^{-1} x\right) d \mu(x)=\int \chi_{E}(x) J_{t}(x) d \mu(x) .
$$

This equality holds for every Baire set $E$ and thus it holds also with any function $f \in B_{+}(G)$ in place of $\chi_{E}$.

Theоrem C. Jacobians satisfy, for every $s, t$,

$$
J_{s t}(x)=J_{s}(t x) J_{t}(x)
$$

for almost all $x$.

Proof. Let $E$ be a Baire set. We have, by $(1), \mu(s t E)=\int_{E} J_{s t}(x) d \mu(x)$. On the other hand, also by (1),

$$
\begin{aligned}
\mu(s t E) & =\int_{t E} J_{s}(x) d \mu(x)=\int \chi_{E}\left(t^{-1} x\right) J_{s}(x) d \mu(x) \\
& =\int_{E} J_{s}(t x) J_{t}(x) d \mu(x) .
\end{aligned}
$$

Since $E$ is arbitrary, the result follows by comparing the integrals. 
Theorem D. If $\mu(E)=0$, then $\mu(E t)=0$.

Proof. The result follows by the stability of $\mu$ if we show that, if $\mu(E)>0$, then $\mu\left(E^{-1}\right)>0$. Then from $\mu(E)=0$ we have $\mu\left(E^{-1}\right)=0$; hence $\mu\left(t^{-1} E^{-1}\right)=0$ and thus $\mu(E t)=0$.

Suppose that $\mu(E)>0$. Let us show that then

$$
I=\int \mu\left(t E^{-1} \cap E\right) d \mu(t)>0 \text {. }
$$

We have

$$
\begin{aligned}
I & =\iint \chi_{E^{-1}}\left(t^{-1} x\right) \chi_{E}(x) d \mu(x) d \mu(t) \\
& =\int_{E} d \mu(x) \int \chi_{E^{-1}}\left(t^{-1} x\right) d \mu(t) .
\end{aligned}
$$

Applying (1) to the last integral, we have, by Theorem $\mathrm{B}$, for each $x$,

$$
\int \chi_{E^{-1}}\left(t^{-1} x\right) d \mu(t)=\int \chi_{E^{-1}}\left(\left(x^{-1} t\right)^{-1}\right) d \mu(t)=\int \chi_{E^{-1}}\left(t^{-1}\right) J_{x}(t) d \mu(t)=\int_{E} J_{x}(t) d \mu(t)>0
$$

So we have (2), by (3) and (4). Now, by (2), there is a $t \in G$ such that $\mu\left(t E^{-1}\right)>0$ and thus $\mu\left(E^{-1}\right)>0$. This completes our proof.

\section{Proof of the main theorem.}

LEMMA 1. Let $m$ be the right invariant Haar measure. There exists a Baire measure $v$ on $G \times G$ and a positive function $J(t, x) \in B(G \times G)$ such that, for any Baire sets $D, E$,

$$
\nu(D \times E)=\int_{D} \mu(t E) d m(t)=\int_{E} d \mu(x) \int_{D} J(t, x) d m(t) .
$$

If $D$ is bounded, then the measure $\mu_{D}(E)$ defined by $\mu_{D}(E)=\nu(D \times E)$ is finite on compact sets. If $m(D)>0$, then $\mu$ and $\mu_{D}$ are equivalent.

Proof. Consider the space $G \times G$ with the Baire measure $m \times \mu$. Define, for $M \subset G \times G$,

$$
v(M)=\int \chi_{M}\left(t, t^{-1} x\right) d(m \times \mu)(t, x) .
$$

For $M=D \times E$ we have, from (6), $\mu_{D}(E)=\int_{D} \mu(t E) d m(t)$. Thus, if $D$ is bounded, then, for compact $E, D E$ is bounded and $\mu_{D}(E) \leqslant m(D) \mu(D E)<\infty$. If $m(D)>0$, then, by the stability of $\mu, \mu_{D}$ and $\mu$ are equivalent.

To define $J(t, x)$, we show that $\nu$ and $m \times \mu$ are equivalent. Since $\mu$ is stable, the functions $P(t)=\int \chi_{M}(t, x) d \mu(x), Q(t)=\int \chi_{M}\left(t, t^{-1} x\right) d \mu(x)$ are, for each fixed $t$, both zero or both positive. Therefore the measures $(m \times \mu)(M)=\int P(t) d m(t), \nu(M)=\int Q(t) d m(t)$ are both zero or both positive.

Applying the Radon-Nikodym theorem to the measures $\nu$ and $m \times \mu$ on $G \times G$, we see that there is a positive function $J(t, x) \in B(G \times G)$ such that 


$$
\nu(M)=\int_{M} J(t, x) d(m \times \mu)(t, x) .
$$

In particular, if $M=D \times E$, we have (5), by (6) and (7). This completes our proof.

We shall use the phrase "for almost all " if the measure concerned is one of the equivalent measures $\mu, \mu_{D}$. It will be convenient to denote a $\mu_{D}$-Jacobian $J_{t}(x)$ by $J_{D}(t, x)$. Given a function $f(x)$ and a set $C \subset G$, we shall say that $f$ is bounded away from zero and infinity on $C$ if there are finite positive constants $c_{1}$ and $c_{2}$ such that $c_{1}<f(x)<c_{2}$ holds for all $x \in C$.

Lemma 2. Let $D \in \mathrm{N}$. The formula

$$
J_{D}(\tau, x)=I_{D}(\tau, x) / I_{D}(e, x),
$$

where $I_{D}(\tau, x)=\int_{D} J(t \tau, x) d m(t)$, defines a $\mu_{D^{-}}$Jacobian. For almost all $x \in G$, the function $J_{D}(\tau, x)$, regarded as a function of $\tau$, is bounded away from zero and infinity on every compact set.

Proof. We have, by (5),

$$
\mu_{D}(\tau E)=\int_{D} \mu(t \tau E) d m(t)=\int_{D_{\tau}} \mu(t E) d m(t)=\nu(D \tau \times E)
$$

because $m$ is right invariant. Thus, again by (5) and by the invariance of $m$,

$$
\mu_{D}(\tau E)=\int_{E} d \mu(x) \int_{D \tau} J(t, x) d m(t)=\int_{E} I_{D}(\tau, x) d \mu(x) .
$$

From (8), for $\tau=e, \mu_{D}(E)=\int_{E} I_{D}(e, x) d \mu(x)$. This can be written in the other form

$$
\int \chi_{E}(x) d \mu_{D}(x)=\int \chi_{E}(x) I_{D}(e, x) d \mu(x) .
$$

Since this equality holds for each Baire set $E$, we deduce easily that it remains valid if we put any function $f \in B_{+}(G)$ in place of $\chi_{E}$. Let, in particular, $f(x)=J_{D}(\tau, x) \chi_{E}(x)$, where $\tau, E$ are fixed. Then, since $I_{D}(e, x)>0$ (cf. Lemma 1),

$$
\int_{E} J_{D}(\tau, x) d \mu_{D}(x)=\int_{E} I_{D}(\tau, x) d \mu(x)
$$

This, together with (8), implies that $\mu_{D}(\tau E)=\int_{E} J_{D}(\tau, x) d \mu_{D}(x)$. Hence $J_{D}$ is a $\mu_{D^{-}}$Jacobian.

To prove the second part of our lemma, observe that, for all compact sets $Q, C, \mu_{Q}(C)<\infty$ by Lemma 1 and hence, by (8) with $\tau=e, D=Q, E=C$, we have that $I_{Q}(e, x)$ is finite for almost all $x \in C$. Since $G$ is a union of a countable increasing sequence $\mathbf{Q}$ of compact sets, almost every $x \in C$ has the property that $I_{Q}(e, x)$ is finite for each $Q \in \mathbf{Q}$, and hence for every compact set. Since $C$ is arbitary, almost every $x \in G$ has this property.

Suppose that $I_{Q}\left(e, x_{0}\right)<\infty$ for each compact $Q$. Let $C$ be compact and let $Q$ be a compact set containing $D C$. Then, for $\tau \in C$,

$$
I_{D}\left(\tau, x_{0}\right)=\int_{D \tau} J\left(t, x_{0}\right) d m(t) \leqslant \int_{Q} J\left(t, x_{0}\right) d m(t)=I_{Q}\left(e, x_{0}\right)<\infty
$$


Hence $I_{D}\left(\tau, x_{0}\right)$ is bounded above on $C$. To prove that $I_{D}$ is bounded below by a positive constant, consider a set $V \in \mathbf{N}$ such that $V V^{-1} V \subset D$. Let $\left\{V t_{1}, \ldots, V t_{n}\right\}$ be a maximal disjoint family of sets of the form $V t_{r}$ which are contained in $D C$ (this family is finite since $D C$ is bounded). For every $\tau \in C, D \tau$ contains at least one $V t_{i}, i \leqslant n$. To see this we note that $V_{\tau}$ cannot be disjoint to all $V t_{i}$, by the above maximality condition. So, for some $i, V \tau \cap V t_{i} \neq \emptyset$, $t_{i} \in V^{-1} V \tau$ and $V t_{i} \subset D \tau$. Since $J\left(t, x_{0}\right)>0$, we have, for each $\tau \in C$,

$$
I_{D}\left(\tau, x_{0}\right)=\int_{D \tau} J\left(t, x_{0}\right) d m(t) \geqslant \int_{v_{i}} J\left(t, x_{0}\right) d m(t)=I_{i}>0
$$

for a certain $i \leqslant n$. Hence $I_{D}\left(\tau, x_{0}\right) \geqslant \min \left\{I_{1}, \ldots, I_{n}\right\}>0$. So we see that $I_{D}\left(\tau, x_{0}\right)$ is bounded away from zero and infinity on $C$ and thus the same is true for $J_{D}\left(\tau, x_{0}\right)$.

This completes the proof of Lemma 2.

We note that from Lemma 2 and Theorem $\mathrm{C}$ we have, for every $s, t$,

$$
J_{D}(s t, x)=J_{D}(\varepsilon, t x) J_{D}(t, x)
$$

for almost all $x$.

LEMMA 3. There is an $x_{0} \in G$ such that $J_{D}\left(t x_{0}^{-1}, x_{0}\right)$, considered as a function of $t$, is bounded ausay from zero and infinity on every compact set and moreover, for almost all s,

$$
J_{D}\left(s t x_{0}^{-1}, x_{0}\right)=J_{D}(s, t) J_{D}\left(t x_{0}^{-1}, x_{0}\right)
$$

holds for almost all $t$.

Proof. A subset $E$ of a measure space $X$ will be called almost equal to $X$ if $X-E$ has measure zero. Let $X, Y$ be measure spaces and let $X \times Y$ be the product space with the product measure. For $E \subset X \times Y, E(x)$ denotes the section of $E$ determined by $x \in X$, i.e. the set of all $y \in Y$ such that $\langle x, y\rangle \in E$. It follows from Fubini's theorem (cf. [1], $\S 36$, Theorem A, p. 147) that $E$ is almost equal to $X \times Y$ if and only if, for almost all $x \in X, E(x)$ is almost equal to $Y$.

Let $M \subset G \times G \times G$ be the set of all triples $\langle s, t, x\rangle$ satisfying (9). Since, for every $s, t$, (9) holds almost everywhere, $M(s, t)$ is almost equal to $G$ and thus $M$ is almost equal to $G^{3}=G \times G \times G$. Consequently, for almost every $x, M(x)$ is almost equal to $G^{2}=G \times G$. Applying Lemma 2, we see that there is an $x_{0}$ such that $J_{D}\left(t, x_{0}\right)$ is bounded away from zero and infinity on every compact set and moreover $M\left(x_{0}\right)$ is almost equal to $G^{2}$. Using again Fubini's theorem, we deduce that almost all $s$ have the property that $M\left(s, x_{0}\right)$ is almost equal to $G$. But then, by Theorem $\mathrm{D}, M\left(s, x_{0}\right) x_{0}$ also is almost equal to $G$ and we have, for almost all $t, t \in M\left(s, x_{0}\right) x_{0}, t x^{-1} \in M\left(s, x_{0}\right)$. This means that (9) holds, with $x=x_{0}$ and $t x_{0}^{-1}$ in place of $t$. Hence (10) follows and Lemma 3 is proved.

We are now in position to complete our proof. We define on $G$ a measure $\eta$ by

$$
\eta(E)=\int_{E} J_{D}^{-1}\left(t x_{0}^{-1}, x_{0}\right) d \mu_{D}(t) .
$$

We now prove that $\eta$ is left invariant. We denote by $Q$ the set of all $s$ such that (10) holds for almost all $t$. Thus $\mu(G-Q)=0$ by Lemma 3. Let $\tau, E$ be arbitrary. For all $s \in Q$, since $J_{D}$ is a Jacobian,

$$
\eta(s E)=\int x_{E}\left(s^{-1} t\right) J_{D}^{-1}\left(t x_{0}^{-1}, x_{0}\right) d \mu_{D}(t)=\int_{E} J_{D}(s, t) J_{D}^{-1}\left(s t x_{0}^{-1}, x_{0}\right) d \mu_{D}(t)=\eta(E) .
$$


In particular, if $E$ is replaced by $\tau E, \eta(s \tau E)=\eta(\tau E)$ holds for all $s \in Q$. From $\mu(G-Q)=0$ and Theorem $\mathrm{D}, \mu\left(G-Q \tau^{-1}\right)=0$. Hence $Q \cap Q \tau^{-1} \neq \varnothing$ and if $s \in Q \cap Q \tau^{-1}$, then we have $\eta(s \tau E)=\eta(\tau E)$ and also, since $s \tau \in Q, \eta(s \tau E)=\eta(E)$. Thus $\eta(E)=\eta(\tau E)$.

Since $J_{D}^{-1}\left(t x_{0}^{-1}, x_{0}\right)$ is bounded away from zero and infinity on compact sets, $\eta$ is equivalent to $\mu_{D}$ and therefore to $\mu$. We have also that $\eta$ is finite on compact sets and thus it is a Haar measure.

This completes the proof of the main theorem.

\section{REFERENCES}

1. P. R. Halmos, Measure theory (Now York, 1951).

2. A. M. Macbeath and S. Świerczkowski, Measures in homogeneous spaces, Fundamenta Math. 49 (1960), 15-24.

The UnIversity

Glasgow 